\title{
Parental and Child Attitudes Towards Pediculosis are a Major Cause Of Reinfection
}

\author{
Deon V. Canyon", Chauncey Canyon and Sami Milani \\ Office of Public Health Studies, University of Hawaii at Manoa, 1960 East-West Rd, Biomed Building \#T103, Honolulu, \\ HI 96822, USA
}

\begin{abstract}
Pediculosis can elicit considerable emotional distress in the infected and their carers, but the role of attitude in head lice reinfection has not been explored. Failure of head lice control is often attributed to insecticide resistance because human aspects of reinfestation are unknown. This study collected data from 128 teenagers with a history of pediculosis to retrospectively explore attitudes towards head lice. One third of female and two thirds of male teenagers were unconcerned about having head lice. One fifth of parents did nothing about their child's head lice infections, while a few male students did not inform their parents when they had pediculosis. This is the first study on the prevalence of human lice carriers who are a primary cause of head lice reinfection. Medical and public health professions need to understand the social reasons for the failure of insecticide-based head lice control.
\end{abstract}

Keywords: Apathy, pediculosis, reinfection, reservoir, teenager.

\section{INTRODUCTION}

Head lice are a six-legged insect known by the scientific name of Pediculus humanus capitis (De Geer) and infection in humans is called pediculosis. Head lice are very common in all countries around the world and they are spreading because of increased travel and resistance to insecticides $[1,2]$.

Pediculosis elicits great alarm among adults that is out of proportion to their medical significance [3,4]. They can be a source of amusement or stigma for the uninfected and an embarrassing nuisance to the infected [5]. Head lice and human beings have evolved together partly because head lice depend totally on humans for their existence. This is a very old relationship since preserved head lice have even been found in the hair of mummies from Egypt buried 9000 years ago [6]. This pest has evolved closely with humans over time and now cannot survive on the blood of any other animal host.

Parison and Canyon (2010) collected data on pediculosis knowledge and attitudes [7]. They found that parents and carers focused on experiences while head lice treaters focused on control issues. The dominant themes that emerged from this study included concerns about treatment products, issues with treating children, blaming others for reinfection, stigma and social issues. The latter two mental health themes represent difficult aspects of pediculosis management, but have largely been ignored by researchers [8].

The stigma 'inherited' from older generations concerned with fatal louse-borne infections appears to be diminishing in younger generations. The concern is that this will lead to

*Address correspondence to this author at the Office of Public Health Studies, University of Hawaii at Manoa, 1960 East-West Rd, Biomed Building \#T103, Honolulu, HI 96822, USA; Tel: (808) 956-6263;

E-mail: dcanyon@hawaii.edu diminishing concern for lice presence and will lead to greatly enhanced transmission rates. In fact, the prevalence we have already observed all over Australia of $10-40 \%$ and globally in all schools may already be a result of this emerging attitude [1,9]. Prevalence of pediculosis significantly varies by country, region, school and classroom. One large and well-conducted study on 135 classrooms throughout the state of Victoria in Australia reported an average infection rate of $13 \%[10]$. It found that commonly assumed risk factors, such as long hair, living in a rural area and age, were not associated with active infection.

Two issues remain fundamental to controlling head lice resistance and reinfection. Resistance can be addressed by modifying treatment protocols, but reinfection is a behavioral and a social problem that requires more research. We already know that certain key individuals (school students) serve as potent carriers who continually reinfect associates [11]. They maintain lice infections due to greater susceptibility, apathy, lack of treatment or lack of awareness due to lack of symptoms.

However, when children are infected, there are always two sides to the infection equation - parents and children. While the behavior of an infected child is often considered primarily responsible for transmission, parental or career behaviors can also have a large impact. Therefore, this study aimed to gain insight into the experiences and attitudes of teenagers who recalled having lice infections to learn what they thought about their infections and their parent's attitudes towards pediculosis.

\section{METHODS}

A multicultural school in Perth, Western Australia with approx. 1700 students from grades 8 to 12 agreed to allow this study to take place on 480 middle-school students in grades 8 and 9 . Grade 7 is the last primary school grade in 
Australia and head lice infections predominantly occur in primary school grades 1 through 6 [1,2]. Ethics approval H2954 was obtained from James Cook University. Consent letters and information sheets were sent home to parents along with a hard copy of the survey. Parents were asked to complete the consent form and return it to the school. Eligible teenagers with parental consent were asked by their parents to complete an anonymous survey and return it to the school. Completed consent forms and surveys were collected from the school for storage and analysis. Participation was thus based on voluntary self-selection. This sampling strategy combines criterion based and convenience approaches, since the targeted participants are those who have direct experience coping with a head lice infestation (criterion) and they are accessible (convenience) [12].

The questionnaire requested basic non-identifying demographic information (grade, gender and hair details) followed by four open-ended questions:

1. When you think about the last time you had head lice, how did you feel?

2. Why do you think you felt that way?

3. How did your parents feel and behave when they found out you had head lice?

4. Why do you think they felt and behaved that way?

Only students who could recall being infected with head lice were included in the study. The anonymous responses were analyzed for salient themes that addressed the questionnaire focus using the computer software package SPSS 21. Cross-tabulations with Chi-square Tests were employed but since $20 \%$ of the frequencies were usually less than 5, this test was not applicable. Thus Goodman and Krustal tau/Uncertainty Coefficient Tests were performed.

\section{RESULTS}

The response rate was good with 133 students out of 480 (27.7\%) participating in the study and 128 surveys that were sufficiently complete to enable analysis. These students had a history of head lice infection and were willing to volunteer to be in the study. The temporal proximity of participants to the infection event and the nature of the event being recalled indicate that recall would have been reasonably accurate. While adult retrospective reports of adverse childhood experiences involve a substantial rate of false negatives, false positive reports are rare [13]. Only $9^{\text {th }}$ graders reported on $8^{\text {th }}$ Grade infections (Fig. 1).

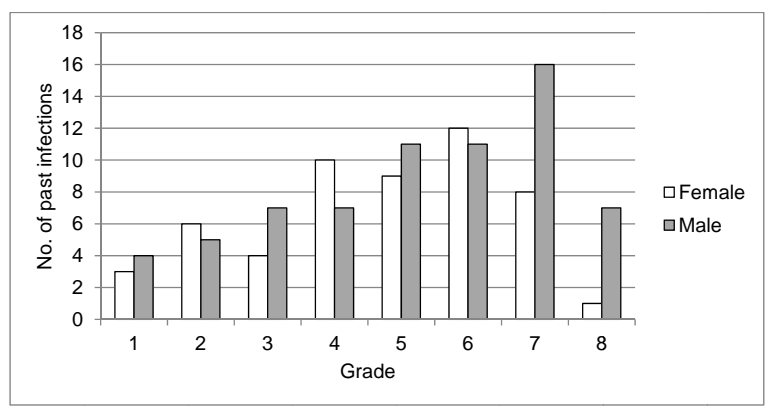

Fig. (1). Number of students who recalled having a head lice infection and the grade in which that infection occurred.
Overall there were $45.3 \%$ females and $53.1 \%$ males while $3 \%$ did not indicate gender (Table 1). There were two Aboriginal females and one African male, but these were omitted from the analysis because there were too few to represent an ethnic background. This left a total of 128 valid questionnaires for Caucasians and Asians.

Table 1. Study Participants by Gender and Race

\begin{tabular}{|c|c|c|c|}
\hline \multirow{2}{*}{ Gender } & \multicolumn{2}{|c|}{ Ethnicity } & \multirow{2}{*}{ Total } \\
\cline { 2 - 3 } & Caucasian & Asian & \\
\hline \hline Female & 50 & 7 & 57 \\
\hline Male & 57 & 10 & 67 \\
\hline Missing & - & - & 4 \\
\hline Total & & & 128 \\
\hline
\end{tabular}

In response to the question, 'What grade are you in now?' there were 66 responders in Grade 8, 59 in Grade 9 and 3 did not respond. Thus the dataset was well distributed between the two grades. In response to the questions about hair thickness, $17.2 \%$ had thin hair, $50.0 \%$ had medium and $32.8 \%$ had thick hair. Blonds made up $16.4 \%$ of the data while $67.2 \%$ had brown hair and $16.4 \%$ had black hair. Shorthaired participants made up $46.4 \%$ of the survey population, $28.0 \%$ had medium hair and $25.6 \%$ had long hair. Short brown medium thickness hair was the most common hair type. This data was unbalanced with only one female and 57 males having short hair, while 28 females and eight males had shoulder length hair, and 29 females and two males had long hair.

\section{Question 1. When You Think About the Last Time You had Head Lice, How Did You Feel at the Time?}

More than half of the total respondents (53.6\%) did not care that they had head lice and most of these were males. A quarter said that they were disgusted or angry about having head lice $(26.4 \%)$. Females made up most of the $7.2 \%$ who were embarrassed and the $12.8 \%$ who were scared or worried about being infected (Fig. 2). When these data were statistically tested using Pearson chi-square and uncertainty coefficient analyses, a significant relationship between the responses to the question and the gender was observed

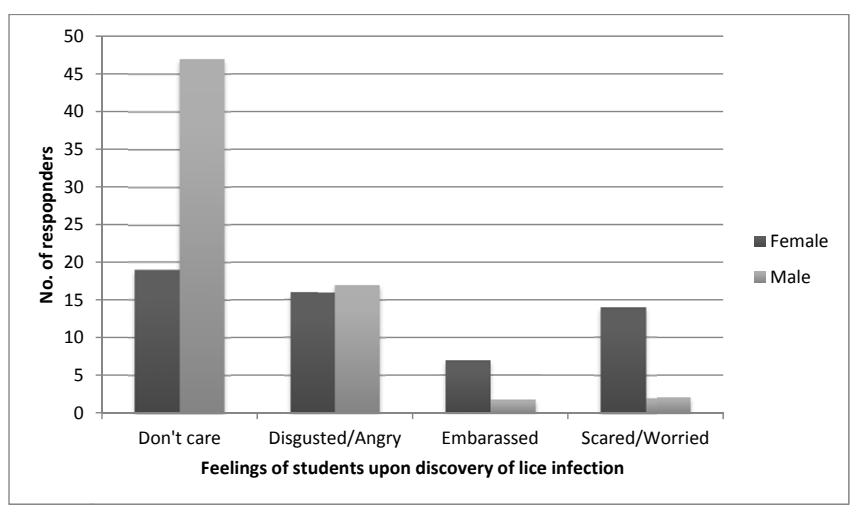

Fig. (2). The range of feelings students had when they found out they had head lice. 
$(24.62, \mathrm{p}<0.001)$. This relationship was moderately strong $(\mathrm{u}=0.118)$ and more gender dependent $(\mathrm{u}=0.156)$. Female responses were fairly evenly distributed, but most males did not care at all. When the responses to this question were statistically tested against ethnicity (Caucasian vs Asian), there was no significant difference.

\section{Question 2. Why Do You Think You Felt that Way?}

A quarter of the students gave answers that indicated that they did not know or did not care about why they did not know or did not care at all $(24.8 \%)$, and most of these were males. Very few stated concerns about treatment and these focused on avoidance of painful or smelly treatments (6.4\%). One third of responders cited pain or itch (36.8\%) and another third cited social issues $(32.0 \%)$ (Table 2). When these data were statistically tested using Pearson chi-square and uncertainty coefficient analyses, a significant relationship between the responses to the question and the gender was observed $(12.764, \mathrm{p}<0.01)$. This relationship was weak $(u=0.056)$ and more dependent on gender rather than the question $(\mathrm{u}=0.079)$. There were no significant differences when the responses to this question were statistically tested against race, hair thickness and hair color. However, hair length was significantly associated with attitude (15.051, $\mathrm{p}<0.05)$. This relationship was weak $(\mathrm{u}=0.053)$ and slightly more hair-length dependent $(\mathrm{u}=0.058)$ (Table 2). Shorthaired students were less inclined to care, treatment issues were more common in longhaired students, itch was greater in shorter haired students, and social issues elevated in shorthaired students.

Table 2. Number of Students Expressing Reasons for their Attitudes About Head Lice Infection Compared with Gender and Hair Length

\begin{tabular}{|c|c|c|c|c|c|}
\hline \multirow{2}{*}{$\begin{array}{c}\text { Reasons for } \\
\text { Attitudes }\end{array}$} & \multicolumn{2}{|c|}{ Student Gender } & \multicolumn{3}{c|}{ Student Hair Length } \\
\cline { 2 - 6 } & Female & Male & Short & Shoulder & Long \\
\hline \hline Don't know/care & 7 & 24 & 21 & 6 & 3 \\
\hline Treatment issues & 6 & 2 & 1 & 2 & 5 \\
\hline Painful/ Itchy & 21 & 25 & 18 & 15 & 11 \\
\hline Social Issues & 23 & 15 & 16 & 12 & 12 \\
\hline
\end{tabular}

\section{Question 3. How did Your Parents React When They Found Out You had Head Lice?}

Students indicated that $15.1 \%$ of parents had no reaction upon finding out that their child was infected with lice, $61.1 \%$ of parents treated the infection, $21.4 \%$ were angry or horrified and $2.4 \%$ did not find out about the infection. When the responses to this question were compared with gender, ethnicity, and hair variables, only gender had a statistical effect on the results (Table 3). This effect was significant $(8.867, \mathrm{p}<0.05)$ but weak $(\mathrm{u}=0.049)$ and more gender dependent $(\mathrm{u}=0.060)$. Parents of male students were more inclined to have no reaction (i.e. no treatment) and a few male students concealed their infections from their parents. If it is conservatively assumed that all the angry/horrified parents treated their children, then $83.1 \%$ of parents treated their children with some head lice control method and $16.9 \%$ of infected students remained untreated.
Table 3. Number of Students Indicating Parental Reactions on Discovering Head Lice Infections in their Children

\begin{tabular}{|c|c|c|c|c|c|}
\hline \multirow{2}{*}{ Parental Reactions } & \multicolumn{2}{|c|}{ Student Gender } & \multicolumn{3}{|c|}{ Student Hair Length } \\
\cline { 2 - 6 } & Female & Male & Short & Shoulder & Long \\
\hline \hline Did not do anything & 4 & 14 & 12 & 3 & 3 \\
\hline Treated hair & 42 & 35 & 30 & 24 & 22 \\
\hline Angry/Horrified & 12 & 14 & 13 & 6 & 7 \\
\hline Did not find out & 0 & 3 & 3 & 0 & 0 \\
\hline
\end{tabular}

\section{Why Do You Think Parents Felt that Way?}

The answers to this question mostly repeated the answers to the last question. Students did not know why their parents had no reaction. They stated that their parents treated because treatment was needed. They stated that parents were angry and horrified because they were concerned about transmission. None of these answers were statistically significant.

\section{DISCUSSION}

The aim of this study was to determine if there were any social factors that played an important role in the transmission and control of head lice and the results provided good evidence to show that social factors are involved and that attitude modification should play an essential role in head lice management.

\section{Question 1. When You Think About the Last Time You had Head Lice, How Did You Feel at the Time?}

The female answers were fairly evenly distributed between the response types, but the male answers were mostly 'don't care' or 'angry'. From the results we see that one third of the females and two thirds of the males answered that they were not concerned about having head lice. This may be because they consider head lice to be a normal part of life and they are confident of being able to eliminate them. However, "Success breeds complacency [and] complacency breeds failure" [14], which translates to increased opportunities for head lice to spread. If these students do not care about being infected then there is a good chance that they will delay informing their parents which will delay treatment and increase transmission. Maunder's (1985) comment that embarrassment and secrecy support the continued survival of head lice is not supported by the results in this study since there was little evidence of stigma driven human behavior [15]. It may be that attitudes have changed over time or that they differ between populations rather than that Maunder was incorrect.

Having a strong personal reaction of disgust was quite common and was shown by one third of females and a quarter of males. But almost no males said that they reacted by becoming embarrassed or scared and worried. Males may thus be less concerned about the social implications of having pediculosis. This reaction was a very female reaction in response to finding out that they had head lice. The lack of significant difference between Caucasians and Asians, does not support the speculation that there is a strong cultural basis for the origin of the feelings about lice [7]. However, 
these two populations resided in the same geographic area and may have not been culturally distinct.

These results indicate that female and male children and teenagers often assume the role of head lice carriers since they apathetically allow their parasites to breed, multiply and spread to other heads. It is important to understand the nature of carriers because they increase the number of head lice in a group of socially linked people, they continuously reinfect the people around them, and they infect new people who come into the social group. Males are twice as likely to act as carriers than females and both genders should be targeted with educational material.

\section{Question 2. Why Do You Think You Felt that Way?}

The information gathered to answer this question showed that gender and hair length were the most important variables. Table 2 shows that a quarter of the students did not care to explain why they felt the way that they did, while half the males who previously said that they did not care, now attributed this feeling to a mixture of treatment issues, pain and itch or social concerns. It may be that this half of the initial 'don't care' group are more important carriers and transmitters of head lice. While male and female attitudes were equally motivated by pain/itch, female feelings were more motivated by social issues. This shows that some teenagers are very self-conscious about having pediculosis because of the way their peers think about head lice. Thus even though these teens might not have negative emotions towards head lice, they will try to control their head lice infection to conform with the values of their peer group. Thus strategies to control head lice, especially in females, need to have a strong component of social motivation to be effective.

Hair length was the other variable that was significantly related to this question. A fairly equal number of students with different hair lengths was concerned about pain/itch and social issues, so hair length was only of interest with regard to the 'don't know/care' and 'treatment' groups. Students in the 'don't know/care' group were more likely to have short hair and students in the 'treatment' group were more likely to have long hair. Since the data already shows a large percentage of males in the 'don't know/care' group, it is clear that they comprise most of the shorthair group. Thus the relationship between shorthair and 'don't know/care' is probably not very important. The treatment reason for attitudes was more interesting because it was clear that longhaired students were more concerned about getting treated and this affected how they felt about their last head lice infection. This was most likely due to painful treatment methods such as fine-toothed combing.

\section{Question 3. How Did Your Parents React (How Did They Behave) when They Found Out You had Head Lice?}

There was a large difference in parental reactions depending on the gender of their child, especially in the 'Did not do anything' category. Parents did not do anything (e.g. treat) to a fifth of their male offspring, whereas almost all of their female offspring experienced a more concerned reaction. This group of head lice infected males is left to fend for themselves so it is also possible that parental reactions have trained some male children not to care and not to be concerned about getting head lice. It is possible that this cycles since a lack of embarrassment and worry in male offspring acts to calm the parents who feel okay about doing nothing.

The lower male score for treatment indicates that less infected males are treated. This result, in combination with other results for males, indicates that they may become important carriers and transmitters of head lice. The last interesting result was that some male students actively concealed their infections from their parents. This behavior may be driven by apathy or a desire to avoid a bad parental reaction or an uncaring parental reaction or unpleasant treatment options. These students are prime carrier candidates because they actively avoid revealing that they have pediculosis.

It has been argued that negative social effects and stigma associated with pediculosis create more problems than the infection itself $[8,16,17]$. This study shows that stigma is a parental and societal issue rather than a child or teen issue since only a quarter of teenagers showed negative emotions towards head lice. Thus head lice control strategies and programs that focus on stigma and negative emotional reactions in teenagers will not be effective. Rather, control strategies should focus on identifying and treating potent apathetic human carriers of head lice who are largely responsible for the perpetual reintroduction of head lice into society.

\section{CONFLICT OF INTEREST}

The authors confirm that this article content has no conflict of interest.

\section{ACKNOWLEDGEMENTS}

Declared none.

\section{REFERENCES}

[1] Gratz NG. Human lice: their prevalence, control and resistance to insecticides: a review 1985-1997. Geneva: World Health Organization, Division of Control of Tropical Diseases, WHO Pesticide Evaluation Scheme, 1997.

[2] Speare R, Buettner PG. Head lice in pupils of a primary school in Australia and implications for control. Int J Dermatol 1999; 38: 285-90.

[3] Counahan M. Scratching for answers? Public health aspects of head lice control. PhD thesis. Townsville, Qld: James Cook University, 2006.

[4] Falagas M, Matthaiou DK, Petros IP, Panos G, Pappas G. Worldwide prevalence of head lice. Emerg Infect Dis. 2008; 14: 1493-4.

[5] Hochman D. One louse, ick. two lice, call for help! New York Times. 9 April 2010.

[6] Mumcuoglu KY, Zias J. Head lice, Pediculus humanus capitis (Anoplura: Pediculidae), from hair combs excavated in Israel and dated from the first century B.C. to the eighth century A.D. J Med Entomol 1988; 25: 545-7.

[7] Parison J, Canyon DV. Head lice and the impact of knowledge, attitudes and practices - a social science overview. In: Management and control of head lice infestations. Bremen: UNI-MED Verlag AG, 2010; 103-9.

[8] Parison J, Speare R, Canyon DV. Head lice: The feelings people have. Int J Dermatol 2013; 52(2): 169-71.

[9] Canyon DV, Speare R. Clinical decision support: Dermatology: Pediculosis. Wilmington, DE: Decision Support in Medicine LLC, 2012.

[10] Counahan M, Andrews R, Büttner P, Byrnes G, Speare R. Head lice prevalence in primary schools in Victoria, Australia. J Paediatr Child Health 2004; 40(11): 616-9. 
[11] Canyon DV, Speare R. Head lice transmission and risk factors. In: Heukelbach J, Ed. Management and control of head lice infestations. Bremen: UNI-MED Verlag AG, 2010; 34-40.

[12] Patton MQ. Qualitative research \& evaluation methods, $3^{\text {rd }}$ Ed. Thousand Oaks, CA: Sage, 2002.

[13] Grove A. Untitled. Brainy Quote. Retrieved 30 Jan 2014 from http://www.brainyquote.com/quotes/quotes/a/andygrove471638.ht $\mathrm{ml}$.
[14] Hardt J, Rutter M. Validity of adult retrospective reports of adverse childhood experiences: Review of the evidence. J Child Psychol Psych 2004; 45(2): 260-73.

[15] Maunder B. Attitude to head lice--a more powerful force than insecticides. J R Soc Health 1985; 105(2): 61-4.

[16] Counahan M. A conditioned response to head lice. Melbourne Vic: Department of Human Services; 2002; May 1-4.

[17] Parison J, Speare, R, Canyon, DV. Uncovering family experiences with head lice: The difficulties of eradication. Open Dermatol J 2008; 2: 9-17.

(C) Canyon et al.; Licensee Bentham Open.

This is an open access article licensed under the terms of the Creative Commons Attribution Non-Commercial License (http://creativecommons.org/licenses/ by-nc/3.0/) which permits unrestricted, non-commercial use, distribution and reproduction in any medium, provided the work is properly cited. 